\title{
Behaviour Prediction Framework in System Architecture Development
}

\section{Krešimir Osman ${ }^{1, a}$, Mario Štorga ${ }^{1, b}$, Tino Stanković ${ }^{1, c}$, Dorian Marjanović ${ }^{1, d}$}

${ }^{1}$ Faculty of Mechanical Engineering and Naval Architecture, University of Zagreb, Ivana Lučića 5, 10000 Zagreb, Croatia

akresimir.osman@fsb.hr, ${ }^{b}$ mario.storga@fsb.hr, ${ }^{2}$ tino.stankovic@fsb.hr,

ddorian.marjanovic@fsb.hr

Keywords: behaviour prediction framework, structural complexity management, model predictive control, managing uncertainty

\begin{abstract}
This paper proposes a Behaviour Prediction Framework with an objective to help designers tackling the problem of uncertainty emerging from system architecture and the effects of the uncertain operating conditions. The proposed framework combines structural and dynamic system model. The Design Structure Matrix is applied to model structural arrangements and dependencies between the subsystems. The Model Predictive Control is applied to model the system in discrete and continuous dynamic domains. As the result of the proposed framework, stability analysis of subsystems in interaction become possible and feedback on system architecture could be provided. To test validity of the proposed approach, the test case involving climate chamber with heat regeneration is presented.
\end{abstract}

\section{Introduction}

Design as an activity is based on the principle of generation and testing solution alternatives until they conform to designer's understanding of what has to be designed. At any abstraction level used during the design process two aspects interfere: establishment of the system architecture and evaluation the system behaviour which emerged as the result of the proposed architectural structure $[1,2]$. Thus, the system's performance is dependent on designer's understanding of a design problem including personal beliefs and experience, and on the emergent behaviour of the system which was designed to perform within certain acceptable limits. Achieved by a designer, behaviour of a technical system may reflect only aspects and traits of behaviour modes which can emerge from established system's architecture introducing in such way uncertainty in the respect to the system functionality. In the literature the uncertainty is defined as a state of having limited knowledge where it is impossible to exactly describe existing state or future outcome, more than one possible outcome [3]. From complex system's research area [4] it is known that even a small change into system's architecture can lead to unexpected or even unstable behaviour of the whole system, or likewise that small perturbation of input conditions as unforeseen environmental conditions or modes of use yield in an undesired system's behaviour. In simple cases uncertainty arising from system environment can be handled by estimating the probabilities of such events or can be handled by use feedback to correct for unexpected or incorrectly predicted environment changes.

This work presents Behaviour Prediction Framework which is aimed to provide designer with a feedback about expected behaviour of predefined subsystem architecture in two cases: under the given operating conditions and in a case of their unexpected change. Provided feedback should point out the elements within system architecture which are not able to operate within given parameters thus causing unstable system behaviour. Although the long term research goal is to establish automated feedback between structural and behavioural domains, at the current research stage the transformation between domains is performed manually, as well as resolving the implications of simulation results to system's architecture. 
The background of this research in the following section will present related work which attempt at unify structural and dynamic system models. Third section will provide more detailed description of the behaviour prediction methodology. Section four will provide description of Behaviour Prediction Framework what is followed by a case study with a goal to evaluate applicability of the proposed framework. Discussion on obtained results and conclusions close this paper.

\section{Combining Structural and Dynamic System Models}

There are two meaningful kinds of complexity relevant for the modern technical systems: structural and behavioural complexity. Modelling the system's structure and its dynamics within the same methodology is gaining importance as it improves the system's understanding. Two domains are usually considered as separate issues: structural complexity management models cannot describe the system's behaviour and dynamical system modelling methods cannot be applied to large scale or complex systems as it requires detailed and expensive process to acquire information about all interactions. One of the recent approaches to combine two domains is presented in work of Diepold et al. [6] as a Multi-Dynamic Mapping framework (see Figure 1) between MultipleDomain Matrices [5] and Generalized Hybrid State Model [7].

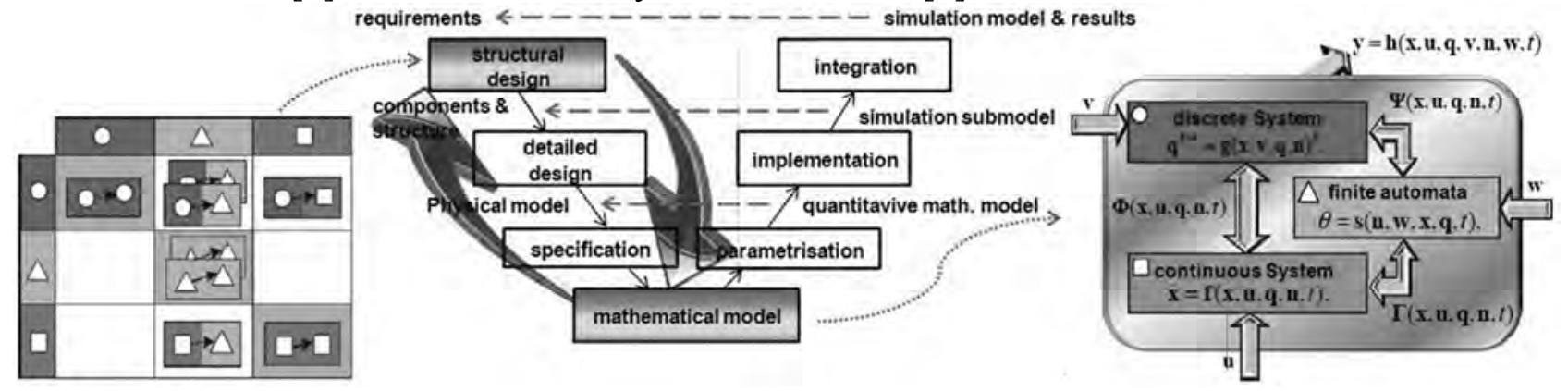

Figure 1. Multi-Dynamic Mapping approach [6]

Multiple-Domain Matrices (MDM) [5] enables different structural views of the system in one model, e.g. requirements, functions, subsystems, components. Method could be used to model the classification of implied domains and dependency types with a goal help designers to keep track of the relevant system aspects and interrelationships. Once all domains are compiled, designers can collect system elements within the domains separately. Such approach allows decomposing, structuring and analysing of the complex systems structural domain.

General Hybrid State Model (GHSM) consists of three dynamical domains [7]: the discrete subsystem, which allows the description of discrete-time variable characteristics between system items, the continuous subsystem allowing continuous-time variable characteristics and the finite automation [8]. The discrete subsystem is thereby given by a classical discrete-time dynamical expression and analogous the continuous subsystem by ordinary differential equations. The finite automation allows a system's theoretical description of system's parts requiring a minimum of knowledge e.g. using Markov chains or Petri nets.

The major interfaces between structural and dynamical system models are constitutionally significant parts of the systems - subsystems that realise key functions. After determining those parts by functional and structural analysis, their behaviour is transformed into a dynamical system representation. The interpretation process transforms the results of the simulation and the findings from the behavioural simulations to answer on the initial problem to be solved. One criterion which is particularly important for the dynamics is the cycle criterion as it is fundamental for feedback loops. Multi-Dynamic Mapping approach use cycles to derive the subsystems, which are significant for the overall system behaviour. However, as there are numerous structural criteria which can be relevant for the system's dynamics, choosing the right criterion for the specific purpose is a critical decision during the structural analysis. 
In example given by Diepold et al. [6], the mapping framework is implemented on very simple mechanical system (the ball-pen) in order to illustrate the potential of the proposed models for adjusting the structural design in consequence of the system's performance. In his work [9], Diepold presented structured process modelling approach by extending the DSM to the DynS-DSM (Dynamical System DSM). The properties of structural analysis are thereby kept up during the whole modelling procedure, which results in a discrete-time representation of the system's dynamics. Structural analysis and the effects of structural changes are thus directly transferable into the system's dynamics supporting a coupled system optimization. DynS-DSM approach is extended to a framework (called quad-I/HS), which supports modelling of hybrid dynamical systems [9].

After the analysis of described work we have identified several drawbacks and defined the following possible extensions:

-In contrast to Diepold's mapping framework [6] our approach should offer possibility to mathematically model all of the subsystems behaviour during the operation time. Our assumption is that all subsystems are linear invariant thus represented in state space model, which is suitable for the further system stability analysis.

-System representation in nonlinear form (as in [6]), omits further possibilities to transform the model into linear form. That will be resolved by using Model Predictive Control (MPC) [10, 11] which allows control and adjustment of working parameters both in discrete and continuous domains thus enabling the modelling of the system in real working conditions. Based on the obtained model, stability analysis of subsystems in interaction becomes possible. Thus, relating the MPC methodology to manage uncertainty is a fundamental contribution and advantage of the presented approach.

- Finally, the improvement of the system is conducted directly within system's dynamical behaviour model, thus reducing the design iteration steps.

\section{Model Predictive Control}

Model predictive control (MPC) is a methodology originating from the process industry denominating a collection of methods which enable control of constrained linear and non linear systems to meet a desired behaviour $[10,11,12]$. Objective in MPC is understood both as the limit to which the system performance is guided to in order to be economically feasible and as an assurance of the performance stability. The former implies that the limit values have to be known a priori, or to be predicted with a degree of uncertainty if necessary, in order to be able to calculate required signals for corrections. The assurance of the performance stability requires that control must provide precise inputs and effects which will be able to meet on-the-fly uncertainties of the performed system behaviour.

In comparison with conventional control methods which try to rectify the outputs based on the feedback provided as a response to actions undertaken, MPC aims at targeting intended (predicted) behaviour. In order to do so, model a discrete time model of the system is utilized to obtain an estimate of its future behaviour.

Estimation of the future behaviour is accomplished by applying a set of input sequences to a model with measured state/output as initial condition, while taking into account imposed constraints. An optimisation problem built around a performance oriented cost function is then solved to choose an optimal sequence of controls from all feasible sequences as close as possible to desired behaviour. To summarise, the MPC is built based on the following principles [10]:

- The explicit use of a process model for calculating predictions of the system behaviour based on the architecture of considered system.

-The optimisations of an objective function subject to constraints, which yields with control trying to maintain system's stability with optimal performance.

- The receding horizon strategy as a goal behaviour which designer expects from the system.

Directing up to prescribed performance values rather than be directed by past behaviour deviations is observed within system design process when experienced designer produces solutions directed by own in-filed knowledge tin order to meet requirements. Search for set of inputs which 
are able to maintain system stability by the MPC can tackle the portion of uncertainties showing to the designer that intended behaviour of the system can be kept stable under imposed conditions. Design process congruent features alongside the MPC's the applicability of multivariable problems and ease of use not requiring in-depth control knowledge qualifies MPC based approach as a strong candidate providing robust behavioural system modelling.

\section{Behavior Prediction Framework}

The proposed Behaviour Prediction Framework (see Figure 2) is aimed to be used during system architecture design phase for structural and behaviour modelling of the systems. We decided to model system structural complexity in only one domain (subsystems domain) in order to point out the importance of the interaction at this level of system abstraction. By using subsystems domain as a generalisation of a more concrete components domain [13], the functional and geometrical complexity of the system may be reduced to a manageable level. The Design Structure Matrix (DSM) $[6,13,14]$ is applied to model arrangements and dependencies between the subsystems. In order to confirm that proposed subsystems arrangements suffice the performance that is expected from the system, the continuous and discrete behaviour domain should be also modelled (see Figure 2) according to general principles of system dynamics.

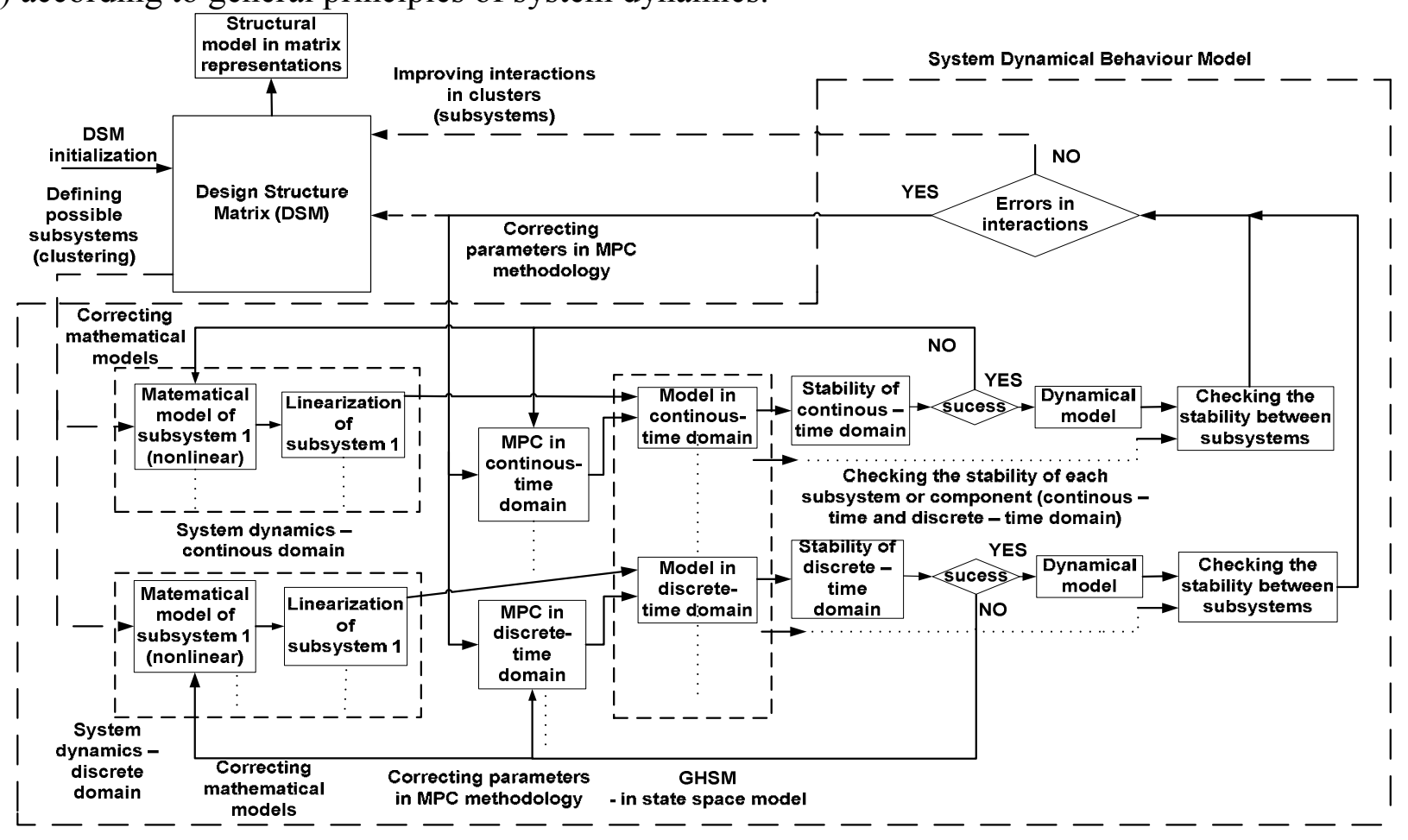

Figure 2. A schema of Behaviour Prediction Framework

The goal of the behaviour modelling is to obtain a linear model in state space representation of the system architecture. The starting point is a non-linear behaviour model of the considered system which may refer to either continuous or discrete time domains. The most common linearization method, which could be applied here, is the expansion in Taylor's series around the equilibrium point [15]. Based on the [16], a high order linear model of dynamical system can be decomposed thus representing it with collections of linear models at different levels of hierarchy. The latter is applied within GHSM [5] module in the framework (Figure 2)

After linearization, the feedback mechanism of the MPC is used to compensate deviations of state variables (system performance) for the predicted equilibrium point. The application of the MPC is to predict the response of the system's output variables relevant for the functionality of the considered system. After applying the MPC the stability is checked for each subsystem for continuous and discrete domain. For energy-based stability analysis Lyapunov method [17] is applied. 
After performance stability is checked for each of subsystems, the result may suggest that some of them are unstable under imposed working conditions. In case of instability, a parameters tuning is performed in mathematical model of the subsystem (e.g. air and water volume flow or other parameters which are in correlation), until the subsystem reaches one of the stable condition states (according to [15]). The next step is to check the stability of subsystems that are in interaction. In case of their instability, a refinement of the system architecture within a DSM is required (e.g. we have to add or remove some entities in system architecture and change their relations in order to improve stability of the interactions). The proposed framework will be further explained and illustrated with a case study of climate chamber with heat regeneration that is described in following section.

\section{Case study - climate chamber with heat regeneration}

The purpose of the case study is to show how Behaviour Prediction Framework can support designers during conceptual design on the example of the climate chamber. Based on the specification of the initial working conditions the simulations of the chamber subsystems behaviour were performed and accordingly to the feedbacks the final system architecture was proposed.

Climate chamber with heat regeneration is very often an integral part of HVAC for large objects (e.g. shopping malls, hotels or business objects). As within energy management (energy cost) the heat regeneration is very desirable goal, fulfilment of the demand for shorter heating/cooling process time in respect to uncertain environmental conditions is very important. For our particular case study initial working conditions for winter period are given as follows: outdoor (environment) temperature $\mathrm{T}_{\mathrm{o}}=-10{ }^{\circ} \mathrm{C}$ (which is average outdoor temperature for town Zagreb, Croatia) and air flow of $\mathrm{q}_{\mathrm{va}}=8,5 \mathrm{~m}^{3} / \mathrm{s}$. Our goal in the case study was to propose architecture for mentioned working conditions and check the stability of the proposed solution for given working conditions. Also, the proposed architecture should be tested to unexpected working conditions in order to simulate the chamber response on the temperature drop assumed at $-30^{\circ} \mathrm{C}$, simulating in such way uncertainty of the working conditions that are stochastically happening in Zagreb area.

The first concept of the chamber architecture was developed based on the designer experience with similar systems as presented on Figure 3. This scheme (for initial working conditions) is a starting point for understanding the relationship between main subsystems. A DSM based on Figure 3 is presented on Figure 4.

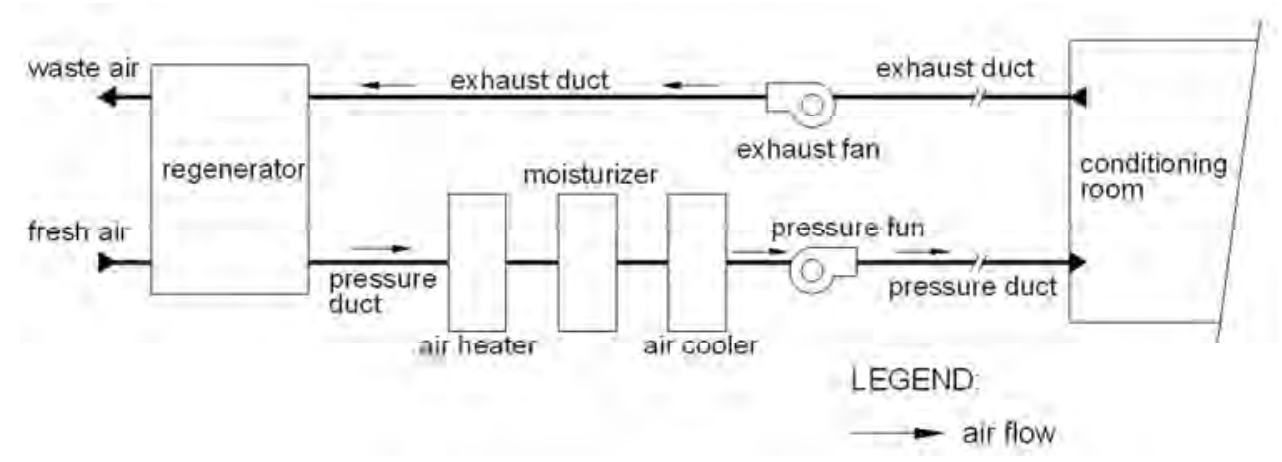

Figure 3. Simplified schema of starting conceptual design of climate chamber

The LOOMEOC (www.teseon.com) was used as a tool for describing climate chamber subsystem structure for further analysis. Possibility for modularization of chamber's subsystems is determined by performing clustering operation over DSM. Figure 4a shows a portion of DSM matrix representation of the architecture after the several steps of refinement including clustering has been conducted. Figure $5 \mathrm{~b}$ presents DSM in graph representation.

Based on the proposed module clustering [13], the behaviour modelling was conducted as follows. First, the initial proposal of the detailed schema for entire system (climate chamber with heat regeneration) was developed (Figure 5). Three subsystems (two air heaters and moisturizer, 
please see Figure 6) were selected for performance stability testing both in response to the initially imposed working conditions $\left(\mathrm{T}_{\mathrm{o}}=-10^{\circ} \mathrm{C}\right)$ and to uncertain working conditions $\left(\mathrm{T}_{\mathrm{o}}=-30{ }^{\circ} \mathrm{C}\right)$. The selected subsystems were chosen because they initiate the highest change of temperature (energy) in heat chamber (from environment in winter period to conditioning conditions).

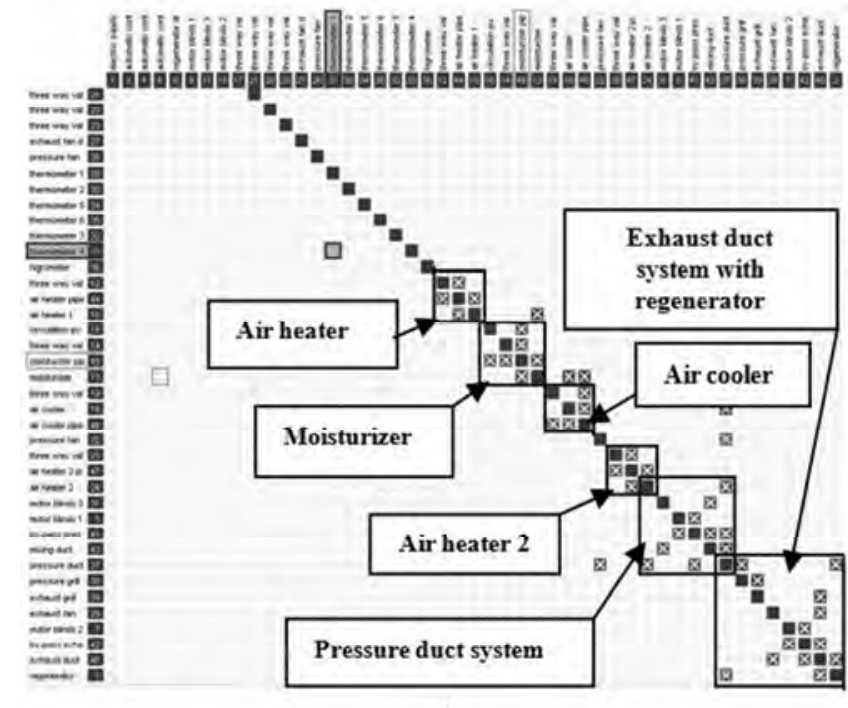

a)

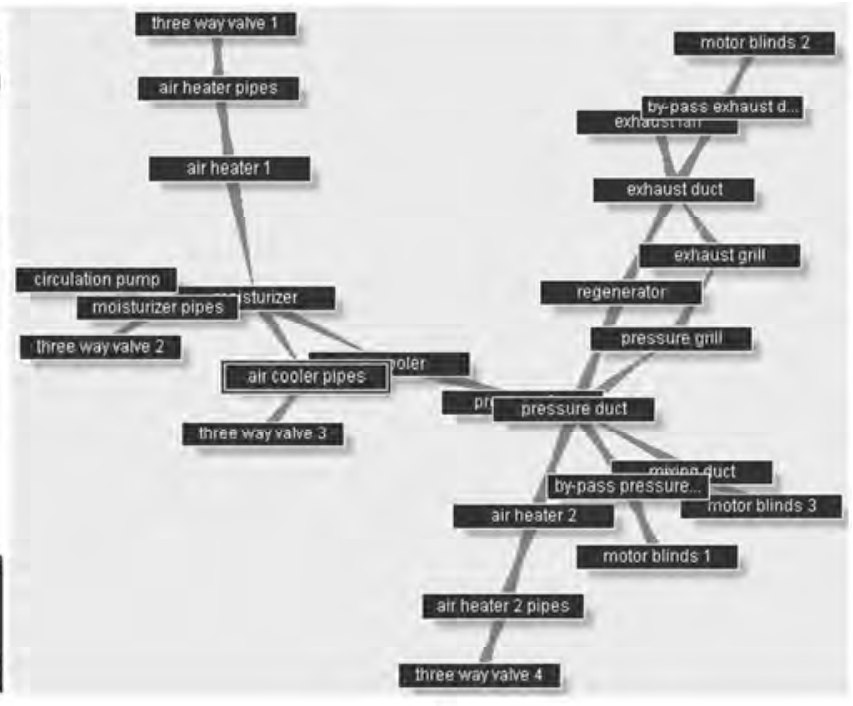

b)

Figure 4. a) Component - based DSM representation with possible modules identified (subsystems), b) Graph representation of system's DSM (screenshots from LOOMEO ${ }^{\circledR}$ )

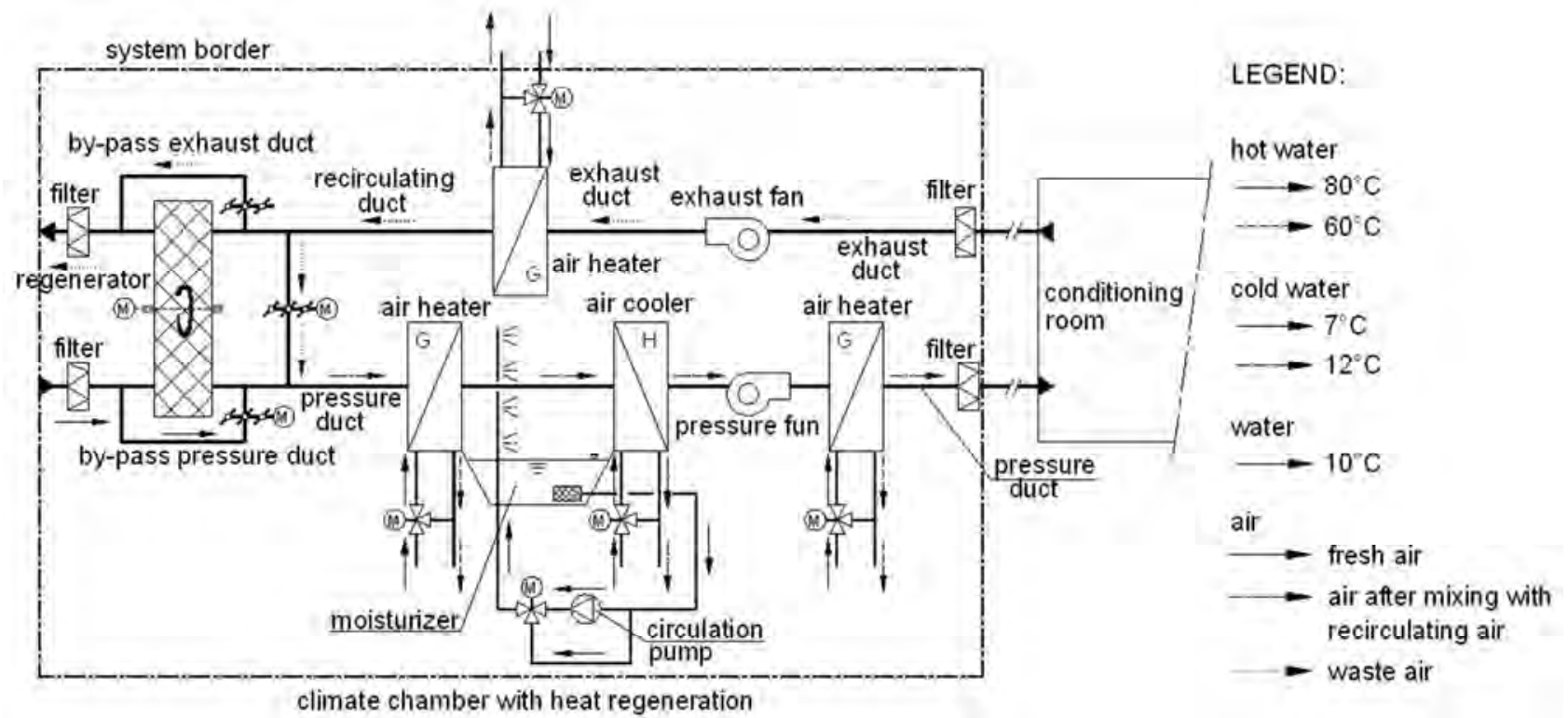

Figure 5. Detail schema of climate chamber with heat regeneration

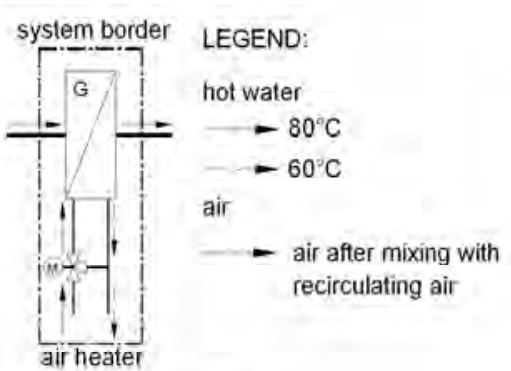

a)

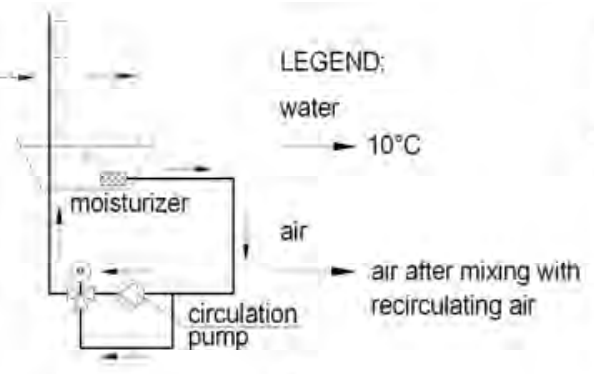

b)

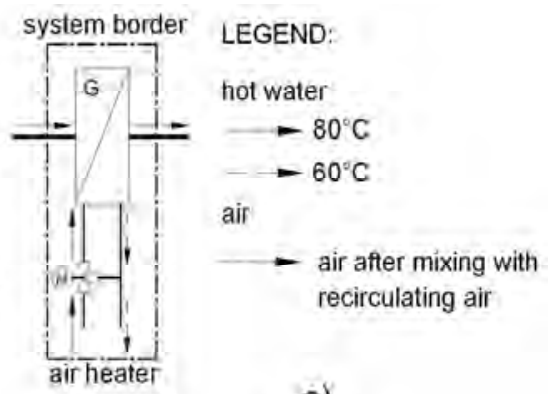

c)

Figure 6. Schema of subsystems: a) subsystem 1 (air heater), b) subsystem 2 (moisturizer), c) subsystem 3 (air heater) 


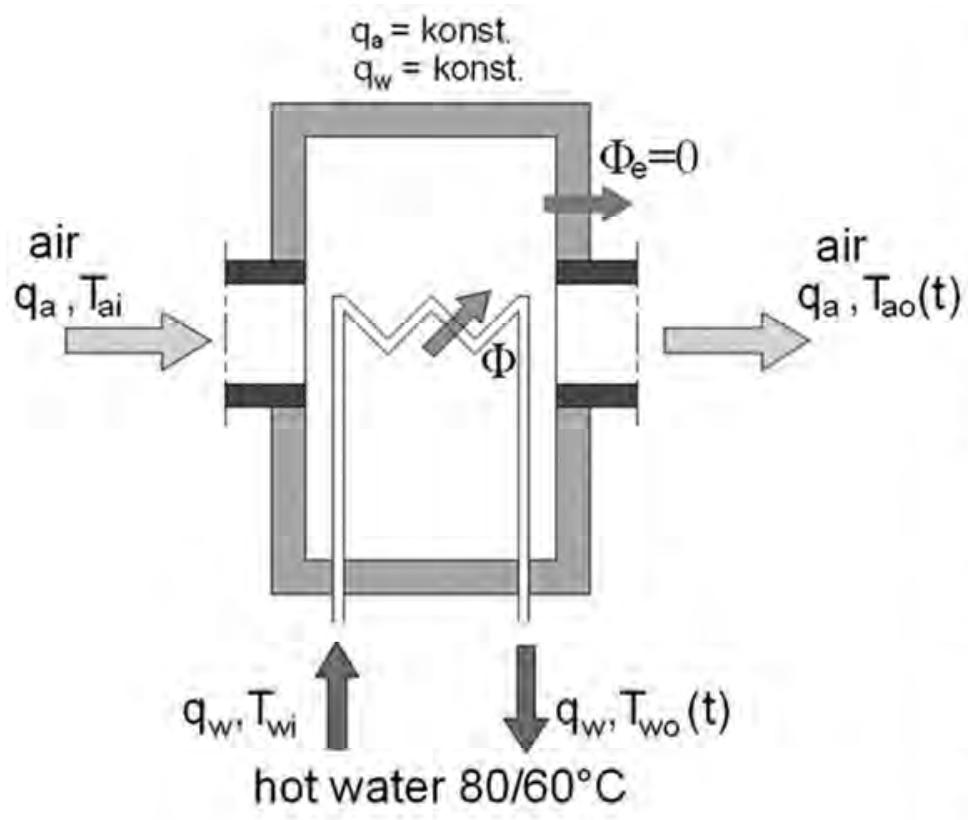

Legend:

$\mathrm{q}_{\mathrm{a}}$ - air mass flow $[\mathrm{kg} / \mathrm{s}] ; \mathrm{q}_{\mathrm{a}}=$ const.

$q_{w}$ - water mass flow $[\mathrm{kg} / \mathrm{s}] ; \mathrm{q}_{\mathrm{w}}=$ const.

$\mathrm{T}_{\mathrm{ai}}$ - air input temperature [K]

$T_{a v}$ - air output temperature $[K] ; T_{a 0}=T_{a b}(t)$

$\mathrm{T}_{w i}$ - water input temperature [K]

$\mathrm{T}_{\mathrm{wo}}$ - water output temperature $[\mathrm{K}] ; \mathrm{T}_{\mathrm{wo}}=\mathrm{T}_{\mathrm{wo}}(\mathrm{t})$

$\mathrm{T}_{b a t}-$ mid barrier temperature $[\mathrm{K}] ; \mathrm{T}_{b \mathrm{~b}}=\mathrm{T}_{\mathrm{bm}}(t)$

$\Phi_{\mathrm{e}}=0 \mathrm{~W}$-thermal flux to environment

$V_{3}\left[m^{3}\right]$ - air volume (in whole subsystem)

$V_{w}\left[m^{3}\right]$ - water volume (in whole subsystem)

$\mathrm{V}_{\mathrm{b}}\left[\mathrm{m}^{3}\right]$ - barrier volume (in whole subsystem)

$\mathrm{c}_{\mathrm{a}}[\mathrm{J} / \mathrm{kgK}]$ - specific heat coefficient for air

$\mathrm{c}_{\mathrm{w}}[\mathrm{J} / \mathrm{kgK}]$ - specific heat coefficient for water

$\mathrm{c}_{\mathrm{b}}[\mathrm{J} / \mathrm{kgK}]$ - specific heat coefficient for barrier

$\rho_{3}\left[\mathrm{~kg} / \mathrm{m}^{3}\right]$ - air density

$\rho_{w}\left[\mathrm{~kg} / \mathrm{m}^{3}\right]$ - water density

$\rho_{\mathrm{b}}\left[\mathrm{kg} / \mathrm{m}^{3}\right]$ - barrier density

$\mathrm{a}_{\mathrm{an}}\left[\mathrm{W} / \mathrm{m}^{2} \mathrm{~K}\right]$ - heat transfer coefficient on air side

$\alpha_{s b b}\left[\mathrm{~W} / \mathrm{m}^{2} \mathrm{~K}\right]$ - heat transfer coefficient on water side

$A_{a t}\left[\mathrm{~m}^{2}\right]$ - heat exchanger area on air side

$A_{\text {wa }}\left[m^{2}\right]$ - heat exchanger area on water side

Figure 7. Simplified model of the subsystem 1

The mathematical model (system of differential equations for energy conservation) for subsystem 1 (air heater - Figure 7) in continuous domain are expressed as follows (1-3):

$$
\begin{aligned}
& \frac{d T_{a o}}{d t}=a_{1} \cdot q_{v, a} \cdot\left(T_{a i}-T_{a o}(t)\right)-a_{2} \cdot\left(T_{a m}(t)-T_{b m}(t)\right) \\
& \frac{d T_{w o}}{d t}=c_{1} \cdot q_{v, w} \cdot\left(T_{w i}-T_{w o}(t)\right)-c_{2} \cdot\left(T_{w m}(t)-T_{b m}(t)\right) \\
& \frac{d T_{b m}}{d t}=b_{1} \cdot\left(T_{a m}(t)-T_{b m}(t)\right)-b_{2} \cdot\left(T_{b m}(t)-T_{w m}(t)\right)
\end{aligned}
$$

where the coefficients $a_{1}, b_{1}, c_{1}, a_{2}, b_{2}, c_{2}$ are given as (4-6):

$$
\begin{aligned}
& a_{1}=\frac{1}{V_{a}}, a_{2}=\frac{\alpha_{a b} \cdot A_{a a}}{\rho_{a} \cdot c_{a} \cdot V_{a}} \\
& c_{1}=\frac{1}{V_{w}}, c_{2}=\frac{\alpha_{w b} \cdot A_{w a}}{\rho_{w} \cdot c_{w} \cdot V_{w}} \\
& b_{1}=\frac{\alpha_{a b} \cdot A_{a a}}{\rho_{b} \cdot c_{b} \cdot V_{b}}, \quad b_{2}=\frac{\alpha_{w b} \cdot A_{w a}}{\rho_{b} \cdot c_{b} \cdot V_{b}}
\end{aligned}
$$

The mid air $T_{a m}$ and water $T_{w m}$ temperature within air heater are given by the following $(7,8)$ :

$$
\begin{aligned}
& T_{a m}(t)=\frac{T_{a i}+T_{a o}(t)}{2} \\
& T_{w m}(t)=\frac{T_{w i}+T_{w o}(t)}{2}
\end{aligned}
$$

All parameters from equations (1-8), depend on the properties of fluids (air and water), material (for air heaters), and on air heater design (dimensions of the case plate for air heater and moisturizer). With presented set of equations (1-8) the mathematical model of the subsystems in state space is defined to be used for behaviour simulation in order to check performance stability. For simulation, the subsystem models were described in external scripts (in MATLAB ${ }^{\circledR}$ editor) and imported to the MATLAB Control System Toolbox ${ }^{\circledR}$, where simulation was performed. The stability was checked with a focus on the internal stability of equilibrium states for homogeneous state equations. 


\section{Discussion}

Figure 8 illustrates the MPC structure overview in MATLAB $^{\circledR}$ MPC toolbox with two manipulated variables (inputs), three outputs and the results for initial and uncertain working conditions. Manipulated variables in the example are: $T_{a i}$ (input air temperature) and $T_{w i}$ (input water temperature). The outputs from subsystem (plant) model are: $T_{a o}$ (output air temperature), $T_{w o}$ (output water temperature) and $T_{b m}$ (middle barrier temperature), which are time dependent variables in our example. Presumed influential factors on the system behaviour for the example in the case study for initial and uncertain conditions are the changes of input temperature to subsystem(s) and air flow though the pressure duct.

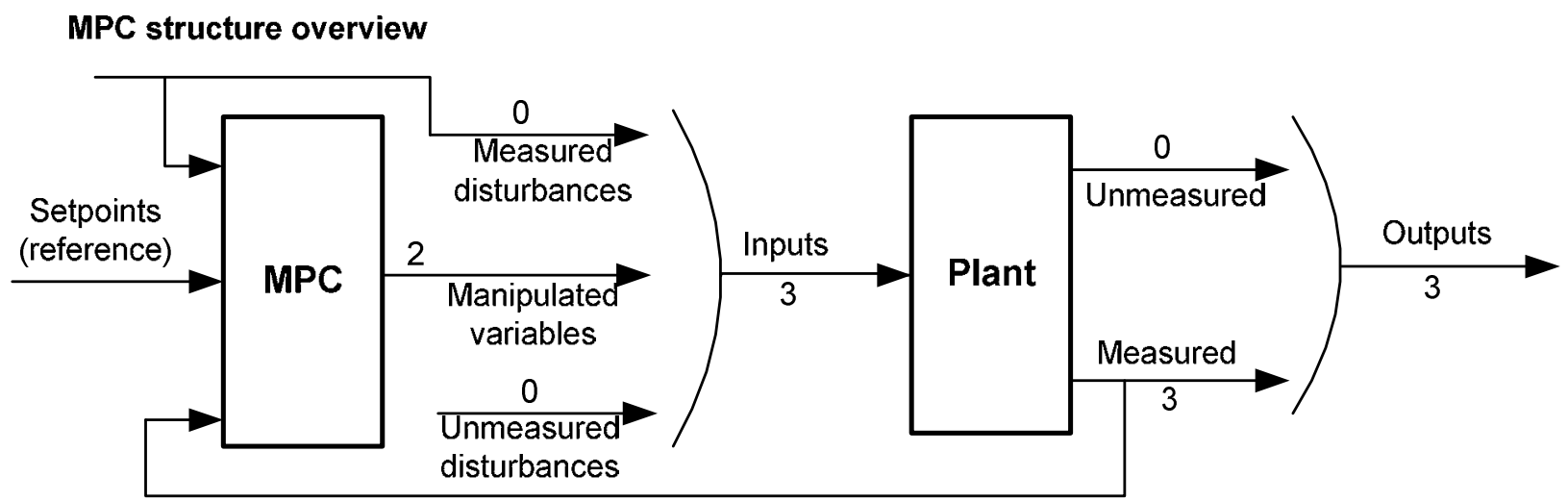

Figure 8. MPC structure overview

The figure 9 depicts simulation outputs for initial working conditions in winter period with input temperature (cca. $\left.T_{o}=-10{ }^{\circ} \mathrm{C}\right)$, and increased air flow $\left(q_{v a}=8,5 \mathrm{~m}^{3} / \mathrm{s}\right)$. On the figure 9a the simulation outputs are shown for improved model after several feedback loops within presented framework were conducted. Improving model assumes change of parameters in mathematical model, adjusting parameters in MPC and changing the system architecture. As said, stability analysis is a part of the proposed framework to check how the considered system will perform under the MPC. The information tells whether the changing and adjusting of parameters was performed in order to reach desired behaviour (i.e. horizon) considering every subsystem and all of the subsystems as a whole. The output architecture is either acceptable or it must be altered to meet the imposed conditions (addition or removal of elements and relations). Figure $9 \mathrm{~b}$ shows subsystem 1 in asymptotic stability (after the few iteration steps) that confirms a suitable solution for subsystem 1 in respect to initial working conditions.

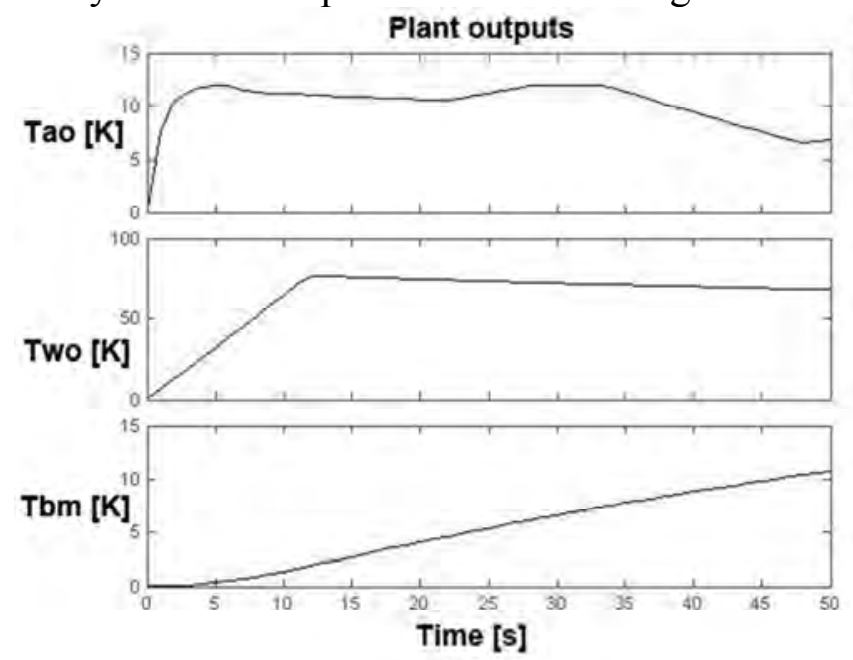

a)

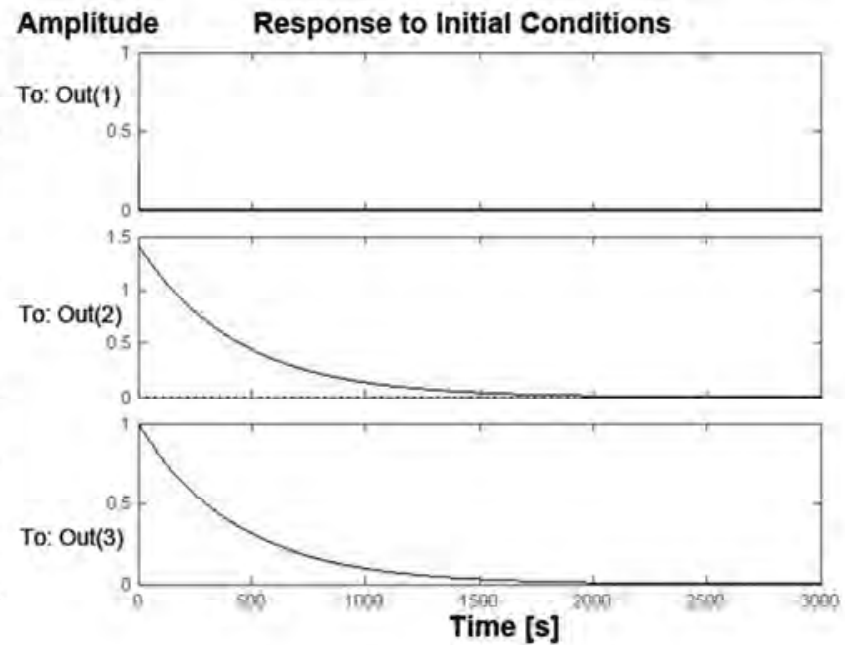

b)

Figure 9. Subsystem 1 at initial working conditions - a) responses in MPC tool, b) stability analysis - asymptotically stable system 
Next step is to repeat the simulation and test system in the unexpected working conditions (outdoor temperature drop to $-30^{\circ} \mathrm{C}$ ) for system architecture derived on account of the previous simulation. Figure 10 shows simulation outputs for unexpected working conditions with input temperature (cca $T_{o}=-30^{\circ} \mathrm{C}$ ). On the figure 10a simulation outputs in MPC are shown for model as derived from the previous simulation (model assumes architecture and process parameters as known). Figure 10b present subsystem 1 in an unstable condition. As not being an acceptable solution for subsystem 1, intervention in mathematical model with parameter adjustment is required.

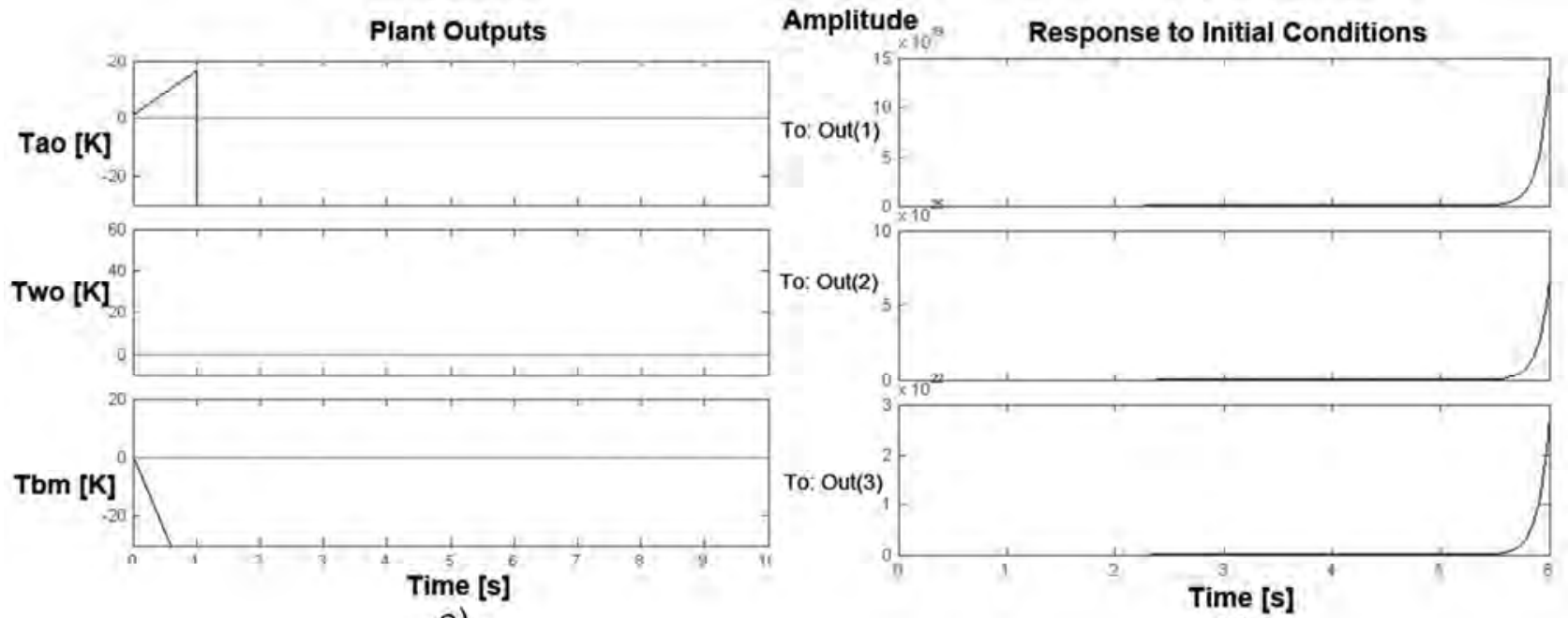

a)

b)

Figure 10. Subsystem 1 at unexpected (uncertain) working conditions - a) responses in MPC tool, b) stability analysis - unstable subsystem

The problem can be resolved by adding these new elements: heat regenerator, recirculation duct and air heater. Every added subsystem assumes a partial role in temperature change thus achieving an increase of the input temperature. Likewise with recirculation duct (and mixing process of fresh air and percent of waste/recirculation air), we decrease air flow throughout the observed system. Starting from the initial system schema (see Figure 3) and refining it throughout proposed framework a climate chamber design concept is obtained which meets both expected and uncertain working conditions.

\section{Conclusion}

The paper proposes a Behaviour Prediction Framework which could help designers with uncertainties of system behaviour and system stability prediction during system architecture development stage. The proposed framework offers opportunity to simplify, improve and accelerate development process for systems that are facing uncertain conditions during operating phase. Based on the proposed simulation and prediction method, it is possible to analyse different system architecture arrangements and subsystems interactions against the changes in architecture elements. Framework also enables designers to make refinement on existing subsystem structures, adding new features to them and predicting new behaviour based on the new features.

Future research will be continued in several directions. One of them should be development of the interface between structural and behaviour model, enabling in such way automatic indication of the problems occurring on subsystems level as the result of dynamical analysis. The other possibility is the research on how to apply the extended MPC involving uncertain evolution sets, i.e. the robust MPC [18]. This could allow consideration of an uncertain system under any admissible uncertainty in order to achieve system robust stability. 


\section{Acknowledgements}

This research is part of funded project "Models and methods of knowledge management in product development" supported by the Ministry of Science and Technology of the Republic of Croatia.

\section{References}

[1] V. Hubka, W. E. Eder: Engineering Design: General Procedural Model of Engineering Design (Springer - Verlag Berlin Heidelberg, Germany, 1992)

[2] C. T. Hansen, M. M. Andreasen: Two approaches to synthesis based on the domain theory, In Engineering Design Synthesis, (ed. A. Chakrabarti), chapter 6, (Springer-Verlag London Limited, UK, 2002), p. $93-108$.

[3] D. Hubbard: How to Measure Anything: Finding the Value of Intangibles in Business (John Wiley \& Sons, USA, 2007)

[4] M. Mitchell: Complexity: A Guided Tour (Oxford University Press, USA, 2009)

[5] U. Lindemann, M. Maurer and T. Braun: Structural Complexity Management - An Approach for the Field of Product Design (Springer - Verlag Berlin Heidelberg, Germany 2009)

[6] K. J. Diepold, W. Biedermann, K. G. M. Eben, S. Kortler, B. Lohmann and U. Lindemann: Combining Structural Complexity Management and Hybrid Dynamical System Modelling, In: Proceedings of DESIGN 2010, Dubrovnik, Croatia, volume 2, (2010), p. 1045 - 1054.

[7] M. Buss, M. Glocker, M. Hardt, O. von Stryk, R. Bulirsch, G. Schmidt: Nonlinear Hybrid Dynamical Systems: Modelling, Optimal Control, and Applications, In: Analysis and Design of Hybrid Systems - Lecture Notes in Control and Information Science (LNCIS), Springer Berlin, Germany, 2002, p. 311-335.

[8] T.A. Helzinger T.A.: The Theory of Hybrid Automata (Berkley, USA, 1996)

[9] K. J. Diepold, F. J. Winkler, B. Lohmann: Systematical hybrid state modelling of complex dynamical systems: The quad - I/HS framework, In: Mathematical and Computer Modelling of Dynamical Systems, Vol. 16, No. 4 (2010), p. 347 - 371.

[10] E.F. Camacho and C. Bordons: Model Predictive Control (Springer - New York, USA 2004)

[11] L. Wang: Model Predictive Control System Design and Implementation Using MATLAB, (Spinger - Verlag London Limited, 2009)

[12] M. Lazar: Model Predictive Control of Hybrid Systems: Stability and Robustness, PhD thesis (Technische Universiteit Eindhoven, Eindhoven, 2006)

[13] D. Steward: The Design Structure Matrix: A Method for Managing the Design of Complex Systems, In: IEEE Transaction on Engineering Management, Vol. 28, No. 3 (1981), p. 321-342.

[14] T. U. Pimmler, S. D. Eppinger: Integration Analysis of Product Decompositions. In: Proceedings of the 1994 ASME-DTM Conference (ASME, Minneapolis, USA, 1994)

[15] R. C. Dorf, R. H. Bishop: Modern Control Systems (Pearson Education, Inc., Upper Saddle River, New Jersey, USA, 2011)

[16] W. Stanislawski, M. Rydel: Hierarchical mathematical models of complex plants on the basis of power boiler example, In: Archives of Control Sciences, Vol. 20, No. 4, (2010), p. 381-416.

[17] A. Bacciotti, L. Rosier: Liapunov Functions and Stability in Control Theory, $2^{\text {nd }}$ edition (Springer - Verlag Berlin Heidelberg, Germany, 2005)

[18] A. Bemporad, M. Morari: Robust Model Predictive Control: A Survey, In: Robustness in Identification and Control, Vol. 245, A. Garulli, A. Tesi, A. Vicino (Eds.), Lecture Notes in Control and Information Sciences, Springer -Verlag (1999), pp. 207-226 
Uncertainty in Mechanical Engineering

doi:10.4028/www.scientific.net/AMM.104

Behaviour Prediction Framework in System Architecture Development doi:10.4028/www.scientific.net/AMM.104.3 